\title{
Fluctuations of Imbalanced Fermionic Superfluids in Two Dimensions Induce Continuous Quantum Phase Transitions and Non-Fermi-Liquid Behavior
}

\author{
Philipp Strack \\ Department of Physics, Harvard University, Cambridge Massachusetts 02138, USA \\ Pawel Jakubczyk \\ Faculty of Physics, Institute of Theoretical Physics, University of Warsaw, \\ Hoża 69, 00-681 Warsaw, Poland \\ (Received 28 November 2013; published 18 April 2014)
}

\begin{abstract}
We study the nature of superfluid pairing in imbalanced Fermi mixtures in two spatial dimensions. We present evidence that the combined effect of Fermi surface mismatch and order parameter fluctuations of the superfluid condensate can lead to continuous quantum phase transitions from a normal Fermi mixture to an intermediate Sarma-Liu-Wilczek superfluid with two gapless Fermi surfaces-even when mean-field theory (incorrectly) predicts a first-order transition to a phase-separated "Bardeen-Cooper-Schrieffer plus excess fermions" ground state. We propose a mechanism for non-Fermi-liquid behavior from repeated scattering processes between the two Fermi surfaces and fluctuating Cooper pairs. Prospects for experimental observation with ultracold atoms are discussed.
\end{abstract}

DOI: 10.1103/PhysRevX.4.021012

Subject Areas: Condensed Matter Physics

\section{INTRODUCTION}

Understanding the nature of strongly correlated fermion systems, which do not obey the rules of Landau's Fermi liquid $[1,2]$, remains one of the most pressing issues in the physics of correlated matter. The unifying property of nonFermi-liquid states are the short lifetimes of quasiparticles. This makes the physics interesting and the theoretical description hard. In strong non-Fermi liquids, there are no well-defined quasiparticles at all; that is, there are no electronic excitations whose lifetime becomes a large time scale when asymptoting energies toward zero. In weak nonFermi liquids, the interactions of fermions with fluctuations leads to reduced lifetimes and anomalous thermodynamics but ultimately well-defined quasiparticles.

Non-Fermi liquids can occur in a variety of physical contexts ranging from ceramic copper oxides and transition-metal compounds at below room temperatures to quantum chromodynamics at finite quark density in conditions similar to those right after the big bang (temperatures of millions of kelvin) [3-6]. A popular and experimentally relevant scenario for non-Fermi-liquid behavior is the proximity to a quantum critical point (QCP) or phase [7,8]. The crossover from Fermi-liquid to non-Fermi-liquid behavior in solid-state materials can be induced, for example, by changing chemical composition, pressure, or straining the crystal lattice.

Published by the American Physical Society under the terms of the Creative Commons Attribution 3.0 License. Further distribution of this work must maintain attribution to the author(s) and the published article's title, journal citation, and DOI.
Until not so long ago, the best known examples of Fermi liquids accessible in tabletop experiments were ${ }^{3} \mathrm{He}[9,10]$ and normal metals with low ordering temperatures. Experiments with ultracold atomic quantum gases [11] provide a complementary route to study interacting Fermi systems, sometimes in novel regimes beyond what is possible in solid-state situations. Using two-body Feshbach resonances, it is now possible to prepare interacting Fermi gases of (electrically) charge-neutral atoms with tunable interactions in odd-integer, nuclear hyperfine states in the quantum degeneracy regime, that is, at nanokelvin temperatures corresponding to fractions of the Fermi temperature [12-14].

Density- and/or mass-imbalanced Fermi mixtures [15-17] represent such a new beyond-solid-state synthetic quantum material. A bold proposition by Liu and Wilczek [18] on pairing in such fermion mixtures, invoking a homogeneous superfluid coexistent with "metallic" fermions on two Fermi surfaces, triggered research on Sarma's earlier proposal [19] as well as various generalization into the modern context of ultracold Fermi gases [20-30].

The common point of departure for these analyses is the attractively interacting (spin-)polarized Fermi gas

$$
\begin{aligned}
H= & \sum_{\mathbf{k} \sigma} \xi_{\mathbf{k} \sigma} c_{\mathbf{k} \sigma}^{\dagger} c_{\mathbf{k} \sigma} \\
& +\frac{g}{V} \sum_{\mathbf{k}, \mathbf{k}^{\prime}, \mathbf{q}} c_{\mathbf{k}+\mathbf{q} / 2, \uparrow}^{\dagger} c_{-\mathbf{k}+\mathbf{q} / 2, \downarrow}^{\dagger} c_{\mathbf{k}^{\prime}+\mathbf{q} / 2, \downarrow} c_{-\mathbf{k}^{\prime}+\mathbf{q} / 2, \uparrow},
\end{aligned}
$$

where the dispersion of the two spin components is $\xi_{\mathbf{k} \sigma}=\left(\mathbf{k}^{2} / 2 m_{\sigma}\right)-\mu_{\sigma}$, with $\sigma=\uparrow, \downarrow$, and $g<0$ is an attractive, structureless contact interaction between the species. Each dispersion is inflection symmetric 
$\xi_{\mathbf{k} \sigma}=\xi_{-\mathbf{k} \sigma} . V$ is the volume and, in general, the masses $m_{\sigma}$ and chemical potentials $\mu_{\sigma}$ of each spin species can be different.

A mean-field diagonalization of Eq. (1) around the static and homogeneous projection of superfluid fermion bilinear containing one fermion from each spin species, $\alpha=\lim _{q \rightarrow 0} \int_{k} \psi_{k+(q / 2) \uparrow} \psi_{-k+(q / 2) \downarrow}$, produces two quasiparticle branches in the superfluid phase

$$
E_{ \pm}=\frac{\xi_{\mathbf{k} \uparrow}-\xi_{-\mathbf{k} \downarrow}}{2} \pm \sqrt{\frac{\alpha^{2}}{2}+\left(\frac{\xi_{-\mathbf{k} \downarrow}+\xi_{\mathbf{k} \uparrow}}{2}\right)^{2}}
$$

where the condition $\xi_{\mathbf{k} \sigma}=0$ defines the (bare) Fermi surfaces of the two species in the normal phase. This quasiparticle energies can have zeros at finite $\alpha$, as shown in Fig. 1, such that the superfluid state can have Fermi surfaces for certain parameters.

Such an exotic superfluid would be an intriguing state of matter: translated to the correlated electron context, it would be a "perfect metal" with large Fermi surfaces and a superconductor at the same time. This is in stark contrast to both conventional (s-wave) BCS superconductors and high-temperature superconductors: in conventional superconductors, the superfluid order parameter gaps out the entire Fermi surface and charge transport occurs exclusively via bosonic Cooper pairs; in the high-temperature superconductors, the gap has a $d$-wave structure leaving nodes on the Fermi surface ungapped, however, with energetically suppressed density of states.

In the Sarma-Liu-Wilczek superfluid, the simultaneous presence of strong phase fluctuations, that is, the Goldstone mode, and the gapless fermions makes this a strongly
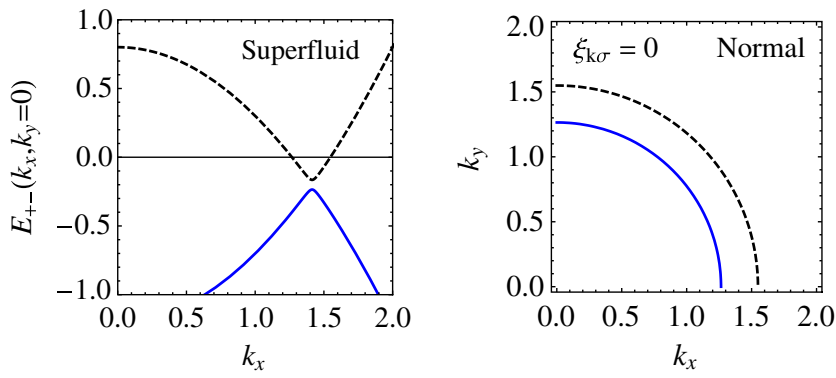

FIG. 1. (Left) Superfluid: Mean-field quasiparticle energies in the superfluid phase. (Right) Normal: Mismatched Fermi surfaces in the normal phase. The smaller, blue Fermi surface is taken to be the $\downarrow$ component, the larger, black-dashed Fermi surface belongs to the $\uparrow$ component. Here and in the rest of the paper, we use $\mu=$ $\left(\mu_{\uparrow}+\mu_{\downarrow}\right) / 2$ as the average chemical potential and $h=\left(\mu_{\uparrow}-\right.$ $\left.\mu_{\downarrow}\right) / 2$ as the average "Zeeman" field. We further define the mass ratio as $r=m_{\downarrow} / m_{\uparrow}$. The Fermi momenta of the two species are given by $k_{F, \uparrow}=\sqrt{2(\mu+h)}$ and $k_{F, \downarrow}=\sqrt{2 r(\mu-h)}$. Here, we use $r=1$ in units of $m_{\uparrow}$ as appropriate for an equal-mass mixture, $\mu=1, h=0.1$, and $\alpha=0.05$. The superfluid "gap" $\alpha$ opens away from the Fermi surfaces [18,19] (where the black-dashed line $E_{+}$ crosses zero). correlated "soup" with anomalous properties that are not fully understood. Endowing such a state with momentum structure in the gap or lattice potentials would complement efforts on high-temperature superconductors where exotic states of matter with (sometime fractionalized) Fermi surfaces are coupled to gapless degrees of freedoms such as gauge bosons.

Parish et al. [24] presented a comprehensive mean-field picture of the imbalanced Fermi gas problem at finite temperatures (focusing on the case of dimensionality $d=3$ ). In particular, they emphasized the possibility of suppressing the temperature of the tricritical point [31] by increasing the mass imbalance between the two fermion species. However, it is rather clear that the picture of Refs. [24,31] is not fully realistic in two spatial dimensions, $d=2$. The first-order (or second-order) transition to the superfluid state at $T>0$ implies the existence of longranged order and, therefore, contradicts the MerminWagner theorem. The important role of fluctuations is also clearly signaled in the recent study [32], operating at Gaussian level. A complete theory accounting for fluctuations should instead (most likely) yield a BerezinskiKosterlitz-Thouless (BKT)-type superfluid involving no true long-ranged order. It is, on the other hand, well known that the BKT transition is actually of infinite order, so that the free energy is of the $C^{\infty}$ class, but not an analytic function. The occurrence of a tricritical point in the phase diagram is, therefore, not admissible, except at $T=0$.

The bottom line of the previous work was that, in the relatively weakly interacting BCS regime of Eq. (1), mismatching the Fermi surfaces typically led to abrupt, first-order Clogston transitions [33] out of the superfluid state into a phase-separated (in real space) state, containing superfluid and normal puddles, which possessed the lowest energy among several candidate states [20-22,24,26]. However, most of these analyses have relied on mean-field theory or focused on three-dimensional Fermi mixtures.

It is worthwhile to note that fluctuation effects are known to change the basic properties of phase transitions in a number of important systems, in low dimensionalities, in particular. It seems that more commonly fluctuations favor the first-order scenario. For a review of the classical systems, see Ref. [34]. On the quantum side, we mention the fluctuation-induced first-order quantum phase transitions in itinerant magnets and superconductors $[35,36]$ and the quantum variant of the Imry-Ma phenomenon recently established rigorously [37]. It was also firmly recognized that a wide class of metallic phase transitions is first order at low temperatures, but (by necessity) second order at sufficiently high $T$ [38].

\section{KEY RESULTS}

This paper addresses the impact of order parameter fluctuations on imbalanced superfluids in interacting Fermi mixtures in two dimensions. Here, we propose the 


\begin{tabular}{|c|c|c|c|c|}
\hline & $\begin{array}{l}\text { Broken } \\
\text { symmetry }\end{array}$ & $\begin{array}{l}\text { Collective } \\
\text { momentum }\end{array}$ & Effective model & $\begin{array}{l}\text { Bare collective } \\
\text { dynamics }\end{array}$ \\
\hline $\begin{array}{l}\text { Spin-density } \\
\text { wave }\end{array}$ & $\begin{array}{l}S U(2) \text { spin } \\
\text { rotation }\end{array}$ & $\begin{array}{l}\text { Commensurate } \\
Q=(\pi, \pi) \text { particle- } \\
\text { hole pair } \\
\text { (Ferromagnet at } \\
Q=(0,0) \text { also possible) }\end{array}$ & $\begin{array}{l}\text { Hot spots on Fermi } \\
\text { surface coupled to } \\
\text { magnon }\end{array}$ & $\begin{array}{l}|\Omega|, z=2 \\
2 \text { Goldstone } \\
\text { modes }\end{array}$ \\
\hline $\begin{array}{l}\text { Nematic Fermi } \\
\text { surface } \\
\text { deformation }\end{array}$ & $\begin{array}{l}\mathrm{C}_{4} \text { lattice } \\
\text { orientation }\end{array}$ & $\begin{array}{l}\text { Forward scattered } \\
Q=(0,0) \text { particle- } \\
\text { hole pair }\end{array}$ & $\begin{array}{l}\text { One Fermi } \\
\text { surface coupled } \\
\text { to photon }\end{array}$ & $\begin{array}{l}|\Omega| /|\mathbf{q}|, \quad z=3 \\
\text { No Goldstone } \\
\text { mode }\end{array}$ \\
\hline \multirow[t]{2}{*}{$\begin{array}{l}\text { Imbalanced } \\
\text { superfluids }\end{array}$} & \multirow[t]{2}{*}{$\begin{array}{l}U(1) \text { number } \\
\text { conservation }\end{array}$} & \multirow{2}{*}{$\begin{array}{l}\text { Homogeneous } \\
Q=(0,0) \text { particle- } \\
\text { particle pair } \\
\text { (LOFF at finite } Q \text { also } \\
\text { possible) }\end{array}$} & \multirow[t]{2}{*}{$\begin{array}{l}\text { Two mismatched } \\
\text { Fermi surfaces } \\
\text { coupled to Cooperon }\end{array}$} & $\begin{array}{l}i \Omega, z=2 \\
1 \text { Goldstone } \\
\text { mode }\end{array}$ \\
\hline & & & & $\begin{array}{l}\text { (Landau damping } \\
\text { also possible) }\end{array}$ \\
\hline
\end{tabular}

FIG. 2. Plain vanilla non-Fermi-liquid "metal" quantum phase transitions at finite fermion density in two spatial dimensions. This plain vanilla classification excludes exotic scenarios such as, e.g., topological order, fractionalization or emergent gauge field, or transitions to fully insulating states. Non-Fermi-liquid properties of the spin-density wave and Fermi surface deformation cases have recently been discussed, for example, in Refs. [39-44]. The interplay with spatially inhomogenous Larkin-OvchinikovFulde-Ferell (LOFF) states is discussed in the text.

possibility of a new type of fluctuation-induced quantum criticality between imbalanced superfluids and a normal Fermi gas as realizable with present-day experimental technology in the relatively weakly interacting BCS regime. It is our hope that the fluctuation-induced quantum criticality of superfluid, charge-neutral Fermi mixtures will become a fruitful addition to the "plain vanilla" set of nonFermi-liquid quantum critical points at finite fermion density tabulated in Fig. 2. To substantiate this claim, here we pursue two complementary directions.

In Sec. III, we present the first computational evidence that strong phase and amplitude fluctuations of the superfluid order parameter suppress the regime of phase separation and lead to a continuous quantum phase transition between an imbalanced Sarma-Liu-Wilczek superfluid with gapless Fermi surfaces and a normal mixture as shown in Fig. 3. This phase diagram is based on the solution of a partial differential (renormalization group flow) equation for the effective potential or free-energy density of the Fermi mixture $U(\alpha)$. The possibility of a quantum phase transition from a state of "2 Fermi surfaces plus BEC" to "2 Fermi surfaces and no BEC" was alluded to in the different microscopic context of mixtures of fermions with fundamental bosons [47]. Because of the fundamental nature of the boson in Ref. [47], this transition does not fall into the plain vanilla non-Fermi-liquid "metal" categorization of Fig. 2, which is restricted to purely fermionic systems.

In Sec. IV, we analyze the diagrammatics of the effective field theory coupling the collective "Cooperon" to two mismatched Fermi surfaces. Non-Fermi-liquid physics appears at two-loop order, despite the fact that the Cooperon reconstructs the fermion dispersions away from the Fermi surfaces. Behind this are repeated scattering processes involving Cooperons and off-shell particle-hole pairs of the respective other species, as shown in Fig. 4.

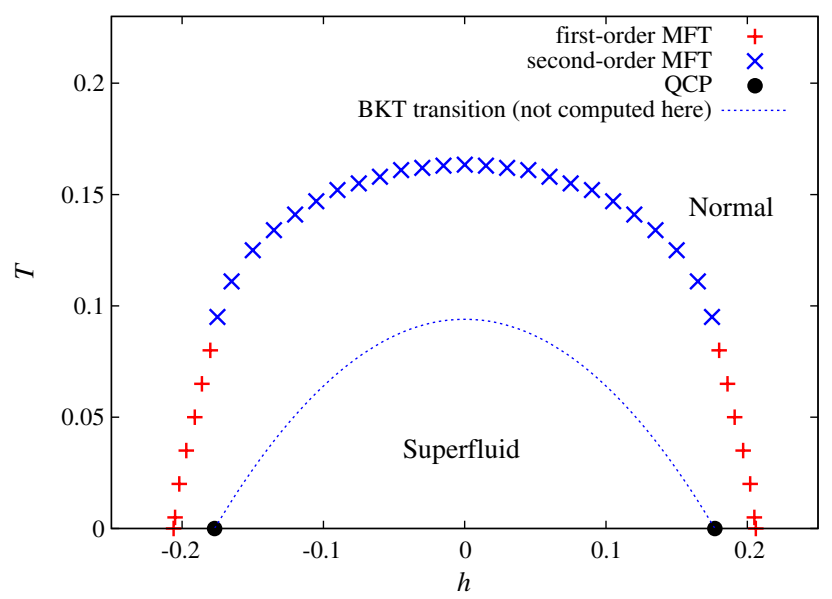

FIG. 3. Fluctuation-corrected phase diagram of imbalanced fermionic superfluids in two dimensions. Goldstone and longitudinal fluctuations induce new quantum critical points (black circles) at $(h= \pm 0.18, T=0)$ between a normal Fermi mixture and a Sarma-Liu-Wilczek superfluid with gapless fermions. Toward $h \rightarrow 0$, another transition to a fully gapped superfluid is expected. Furthermore, fluctuations suppress spontaneous symmetry breaking to $T=0$. Extension of our computations to finite $T$ [45] would lead to a BKT transition line connecting the new QCPs [46]. Mean-field theory (MFT) predicts an incomplete picture: a superfluid dome (blue crosses) with long-range order and first-order transitions on its wings (red crosses).

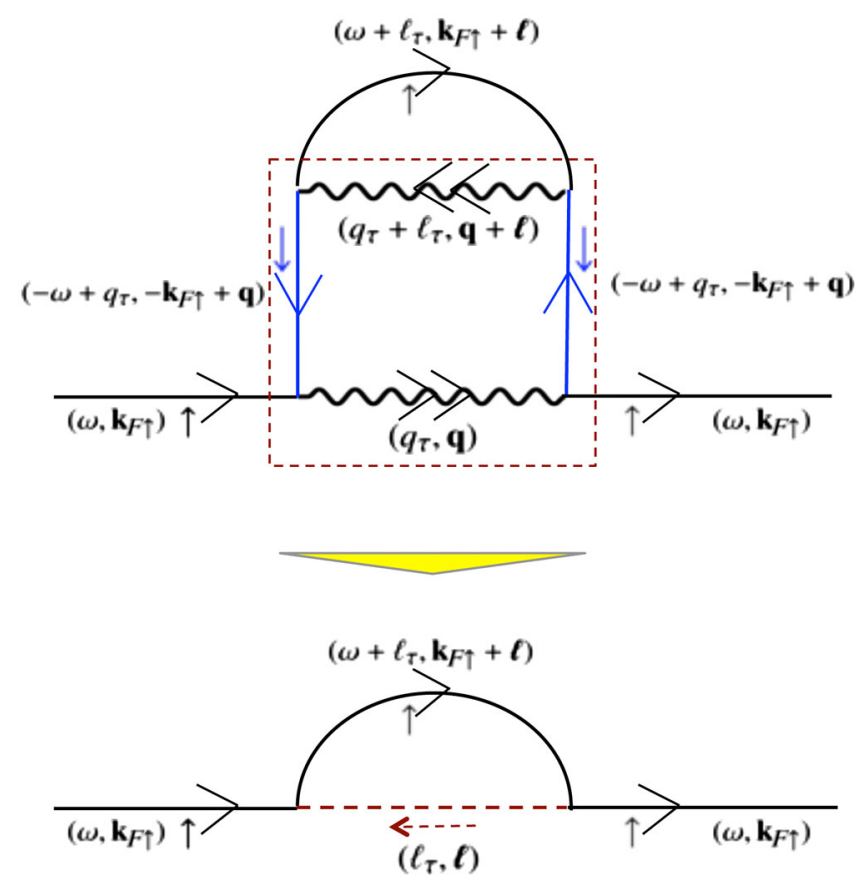

FIG. 4. Repeated scattering of Cooperons and particle-hole pairs of cold, off-shell fermions (red, dashed box in upper graph) mediates a singular, intraspecies interaction (red, dashed line in bottom graph) that induces non-Fermi-liquid behavior on the Fermi surfaces. Wiggly lines here denote the one-loop dressed (cf. Fig. 9) Cooper pair propagator. 
This enhances the scattering rate around the Fermi surface, which should be observable with experimental techniques such as rf spectroscopy $[17,48]$. One may therefore say, on mean-field level, both Fermi surfaces remain "cold" at the superfluid transition. Beyond mean field, however, order parameter fluctuations "warm them up," leading to faster than Fermi liquid decay rates but probably ultimately stable quasiparticles. Different, interesting mechanisms to achieve non-Fermi-liquid features with imbalanced Fermi gases were given in Refs. [49,50]. Putting the attractively interacting Fermi mixture into spin-dependent optical lattices [27], which deform the bare Fermi surfaces such that "band crossing" are generated, also naturally leads to non-Fermi-liquid physics from pairing at least close to the "hot" crossing points.

Our results of Secs. III and IV undoubtedly point out the qualitative importance of fluctuations to the physics of imbalanced superfluids in two dimensions. Like any other quantum critical point for interacting Fermi systems at finite density, however, fluctuations in the vicinity of the QCPs (black dots in Fig. 3) will attract competition from other instabilities. To this end, two important aspects should be investigated further.

The first one is the interplay with induced intraspecies $p$-wave superfluidity $[23,51]$, whose ordering scales, however, are expected to be very small in the relatively weakly interacting BCS regime. In fact, there are similarities to the case of induced $d$-wave pairing close to antiferromagnetic critical points in metals [39,52]. There, singular magnetic fluctuations have two counteracting effects: they reduce the amount of electronic spectral weight around the Fermi surfaces (especially the hot spots), and at the same time, they generate logarithmic singularities in the $d$-wave pairing channel. A similar competition occurs here in imbalanced superfluids: singular Cooper pair fluctuations generate intraspecies pairing tendencies in the $p$-wave channel; at the same time, the lifetimes of quasiparticles around the Fermi surfaces are reduced by the mechanism of Fig. 4 .

The second open question is the interplay with translational symmetry breaking tendencies from inhomogeneous Larkin-Ovchinikov-Fulde-Ferell (LOFF) pairing (see, for example, Refs. [49,53-55] for analysis in one-dimensional systems). As pointed out by James and Lamacraft [49], scattering off singular Cooperons with spatial modulation is a natural route to non-Fermi-liquid behavior because it directly connects the mismatched Fermi surfaces. At the mean-field level, Sheehy and Radzihovsky provided a comprehensive analysis of the imbalanced Fermi gas in the continuum [56] with the result that the inhomogeneous LOFF states occupy only a very narrow strip in the phase diagram. A very recent study [32] emphasizes the prominent role of fluctuations in $d=2$ and suggests that (even at the Gaussian level) the LOFF states are unstable at $T>0$.

In lower dimensionality $d<2$ and under inclusion of lattice potentials, periodic modulations of the phase and or the amplitude of the superfluid order parameter can be stabilized at the mean-field level [57], and an extension of our theory to this case is an interesting future direction. A fluctuation theory beyond Gaussian level for this case is significantly more complicated: in addition to the global (number) charge symmetry, continuous translation symmetry is also broken, leading to an additional Goldstone "phonon" in the fluctuation spectrum. One would then deal with a non-Fermi liquid coupled to a damped, bosonic sector of the $O(4)$ universality class.

We conclude in Sec. V and give a brief outlook on experimental observation with ultracold quantum gases.

\section{FUNCTIONAL FLOW TO CRITICALITY}

For interacting Fermi systems at the brink of phase separating and abrupt jumps of the order parameter (as is the case in the mean-field theory for imbalanced Fermi mixtures at low temperatures), an expansion of the effective potential in powers of the order parameter is not possible. The approach of Ref. [58] proposed to integrate a functional flow equation of the local potential along a continuous flow parameter $\Lambda$ this way capturing order parameter fluctuations keeping the entire local potential on a discrete set of points in field space. The initial conditions for this flow at the scale $\Lambda_{0}$ (the characteristic energy below which collective order parameter fluctuations become important) is given by the mean-field effective potential of Eq. (1):

$$
\begin{aligned}
U^{\Lambda=\Lambda_{0}}(\alpha)= & \frac{-1}{2 g} \alpha^{2} \\
& -T \sum_{k_{\tau}, \mathbf{k}} \ln \left[\frac{\left(-i k_{\tau}+\xi_{\mathbf{k} \uparrow}\right)\left(i k_{\tau}+\xi_{-\mathbf{k} \downarrow}\right)+\alpha^{2} / 2}{\left(-i k_{\tau}+\xi_{\mathbf{k} \uparrow}\right)\left(i k_{\tau}+\xi_{-\mathbf{k} \downarrow}\right)}\right],
\end{aligned}
$$

where $\alpha$ is the real-valued ordering field in the longitudinal $\sigma$ direction in the local, Mexican hat potential that involves all powers of the ordering field. The mean-field theory is derived in Appendix A. The transverse Goldstone quantum field will be denoted by $\pi$. In two dimensions, the attractive interaction $g\left(\Lambda_{\mathbf{k}_{F}}\right)=-\left[2 \pi /\left(m \ln \left[a_{2 \mathrm{D}} \Lambda_{\mathbf{k}_{F}}\right]\right)\right]$ changes logarithmically with momentum scale $[59,60]$, and we will evaluate it at a characteristic scale of the order of the Fermi momenta involved, $\Lambda_{\mathbf{k}_{F}}$. The particle-particle ladder for two particles in vacuum has a logarithmic singularity for small and large momenta. Therefore, two particles form a bound state for arbitrarily weak attraction among them $[61,62]$. Here $a_{2 \mathrm{D}}$ is the 2D scattering length. This paper restricts to the BCS regime, $\left|\Lambda_{\mathbf{k}_{F}}\right| a_{2 \mathrm{D}} \gg 1$, such that gaps are relatively small and we are well separated from the strong-coupling region where the crossover to the BoseEinstein condensation (BEC) regime sets in [60]. We may, therefore, expect BCS-BEC crossoverlike contractions of the Fermi volumes [63] to be subdominant, and we perform 


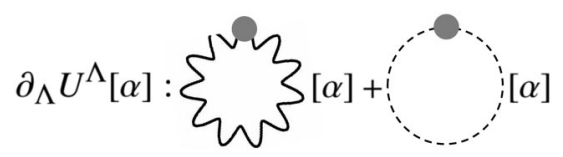

FIG. 5. Graphical representation of the flow equation [Eq. (4)] involving traces over field $(\alpha)$-dependent Goldstone (wiggly loop) and longitudinal (dashed loop) propagators, respectively. Gray dots denote cutoff insertions.

our calculations at constant chemical potentials (using their difference as the tuning parameter).

We now proceed to integrate order parameter fluctuations with the functional flow equation for the effective potential,

$$
\partial_{\Lambda} U^{\Lambda}(\alpha)=\frac{1}{2} \operatorname{tr}_{q}\left\{\partial_{\Lambda} R^{\Lambda}(q)\left[G_{\sigma \sigma}^{R}(q ; \alpha)+G_{\pi \pi}^{R}(q ; \alpha)\right]\right\},
$$

where $\operatorname{tr}_{q}=T \sum_{q_{\tau}} \int d^{2} \mathbf{q} /(2 \pi)^{2}$ stands for frequency and momentum integrations over the field-dependent Goldstone and longitudinal loops shown in Fig. 5. At vanishing cutoff scale $\Lambda \rightarrow 0$, all of the fluctuation modes are incorporated into the partition function and $F(\alpha)=$ $\lim _{\Lambda \rightarrow 0} U^{\Lambda}(\alpha)$ is the free-energy density. Both the longitudinal propagator $G_{\sigma \sigma}^{R}(k ; \alpha)$ and the Goldstone propagator $G_{\pi \pi}^{R}(k ; \alpha)$ are explicitly field $(\alpha)$ dependent

$G_{\pi \pi}^{R}(q ; \alpha)=\frac{Z_{\Omega}\left(\alpha_{0}\right) q_{\tau}^{2}+Z_{\mathbf{q}}\left(\alpha_{0}\right) \mathbf{q}^{2}+m_{\pi \pi}^{2}(\alpha, R)}{\operatorname{det}^{R}(q ; \alpha)}$,

$G_{\sigma \sigma}^{R}(q ; \alpha)=\frac{Z_{\Omega}\left(\alpha_{0}\right) q_{\tau}^{2}+Z_{\mathbf{q}}\left(\alpha_{0}\right) \mathbf{q}^{2}+m_{\sigma \sigma}^{2}(\alpha, R)}{\operatorname{det}^{R}(q ; \alpha)}$.

The "mass" terms for the Goldstone boson $m_{\pi, R}^{2}(\alpha)=$ $U^{\prime}(\alpha)+R^{\Lambda}(q)$ and the longitudinal propagator $m_{\sigma, R}^{2}(\alpha)=$ $U^{\prime}(\alpha)+\alpha^{2} U^{\prime \prime}(\alpha)+R^{\Lambda}(q)$ involve a regulator $R^{\Lambda}(q)$, to be specified below, and are evaluated at general $\alpha$. Here, $U^{\prime}(\alpha)$ is a first- and $U^{\prime \prime}(\alpha)$ is the second-field derivative with respect to $\rho=\frac{1}{2} \alpha^{2}$. The determinant in $(\sigma, \pi)$ field space is

$$
\begin{aligned}
\operatorname{det}^{R}(q ; \alpha)= & {\left[Z_{\Omega}\left(\alpha_{0}\right) q_{\tau}^{2}+Z_{\mathbf{q}}\left(\alpha_{0}\right) \mathbf{q}^{2}+m_{\sigma \sigma}^{2}(\alpha, R)\right] } \\
& \times\left[Z_{\Omega}\left(\alpha_{0}\right) q_{\tau}^{2}+Z_{\mathbf{q}}\left(\alpha_{0}\right) \mathbf{q}^{2}+m_{\pi \pi}^{2}(\alpha, R)\right] \\
& +X^{2}\left(\alpha_{0}\right) q_{\tau}^{2} .
\end{aligned}
$$

The $Z$ and $X$ factors are frequency and momentum renormalization factors and are generally evaluated at the minimum of the effective potential $\alpha_{0}$. In the superfluid phase away from the critical point, $\sigma$ and $\pi$ components of the $Z$ factors can exhibit very different scaling properties in the infrared $[64,65]$. Since our main interest here is the phase diagram and not the infrared asymptotics, here we will keep only one set of $Z$ and $X$ factors. The $X$ factor multiplies a linear time derivative $G_{\sigma \pi}^{R} \sim X q_{\tau}$ and couples the Goldstone and longitudinal fluctuations. In the present renormalization scheme, we supplement only the diagonal components of the inverse propagator with the cutoff term $R$. Within such a procedure, there arises no contribution from $G_{\sigma \pi}^{R}$ in Eq. (4), and the impact of the $X$ factor on the flow of $U$ occurs only via the determinant.

Equation (4) emerges upon evaluating the exact flow equation for the generator of 1-particle-irreducible vertices [66] at a uniform field configuration. The resultant formula involves field- and scale-dependent $Z$ and $X$ factors. The essence of the approximation leading to the closed set of Eqs. (4)-(6) amounts to disregarding these dependencies. An important ingredient of the present study resides in the local potential Eq. (3), which is the starting point for the solution of Eq. (4), and which is directly and clearly related to the microscopic, fermionic system (see Appendix A for a derivation).

Let us also note that Eqs. (4)-(6) fulfill the MerminWagner theorem [67] upon turning on a temperature and provide a framework to study quantum fluctuations beyond BCS theory in a superfluid state without violating Goldstone's theorem, that is, without generating an unphysical Goldstone mass $[65,68,69]$. This is because the flow of the minimum of the potential $\alpha_{0}$ is self-consistently determined from the condition (Ward identity) that the (unregulated) Goldstone mass $m_{\pi, R=0}\left[\alpha_{0}\right]^{2}=U^{\prime}[\alpha] \equiv 0$ vanishes for all $\Lambda$.

We now present our results from solving the functional flow of Eqs. (4)-(6). Using a combined frequency and momentum cutoff $R_{\Lambda}\left(q_{\tau}, \mathbf{q}\right)=Z_{\mathbf{q}}\left(\Lambda^{2}-q^{2}-\frac{Z_{\Omega}}{Z_{\mathbf{q}}} q_{\tau}^{2}\right) \times$ $\theta\left(\Lambda^{2}-q^{2}-\frac{Z_{\Omega}}{Z_{q}} q_{\tau}^{2}\right)$, the frequency and momentum integrations can be performed and Eq. (4) becomes

$$
\begin{aligned}
\Lambda \partial_{\Lambda} U_{\Lambda}(\rho)= & -\frac{\tilde{Z} \Lambda^{2}}{4 \pi^{2}}\left[M_{\sigma \sigma}(\rho)+M_{\pi \pi}(\rho)\right]\left\{-\frac{\tilde{Z} \Lambda}{X^{2}}\right. \\
& +\left(\frac{\Lambda^{2}}{X \sqrt{M_{\pi \pi}(\rho) M_{\sigma \sigma}(\rho)}}+\frac{\tilde{Z}^{2} \sqrt{M_{\pi \pi}(\rho) M_{\sigma \sigma}(\rho)}}{X^{3}}\right) \\
& \left.\times \arctan \left[\frac{\Lambda X}{\tilde{Z} \sqrt{M_{\pi \pi}(\rho) M_{\sigma \sigma}(\rho)}}\right]\right\}
\end{aligned}
$$

with $\rho=\alpha^{2} / 2, \tilde{Z} \equiv \sqrt{Z_{\Omega} / Z_{\mathbf{q}}}$, the regulated longitudinal mass $M_{\sigma \sigma}(\rho) \equiv Z_{\mathbf{q}} \Lambda^{2}+U^{\prime}(\rho)+2 \rho U^{\prime \prime}(\rho)$, and the regulated Goldstone mass $M_{\pi \pi}(\rho) \equiv Z_{\mathbf{q}} \Lambda^{2}+U^{\prime}(\rho)$.

Equation (7) is a second-order partial differential equation, which we solve numerically on a grid in field space containing up to 200 points, where the nonzero minimum (initial) is situated around the middle of the box. The initial condition for $U(\alpha)$ is the untruncated, mean-field effective potential Eq. (3) containing all orders in the order parameter field.

As described in Fig. 3, fluctuations may have a drastic effect on the even qualitative features of the phase diagram. Here, quantum fluctuations suppress the first-order transition and associated phase separation between a BCS plus 

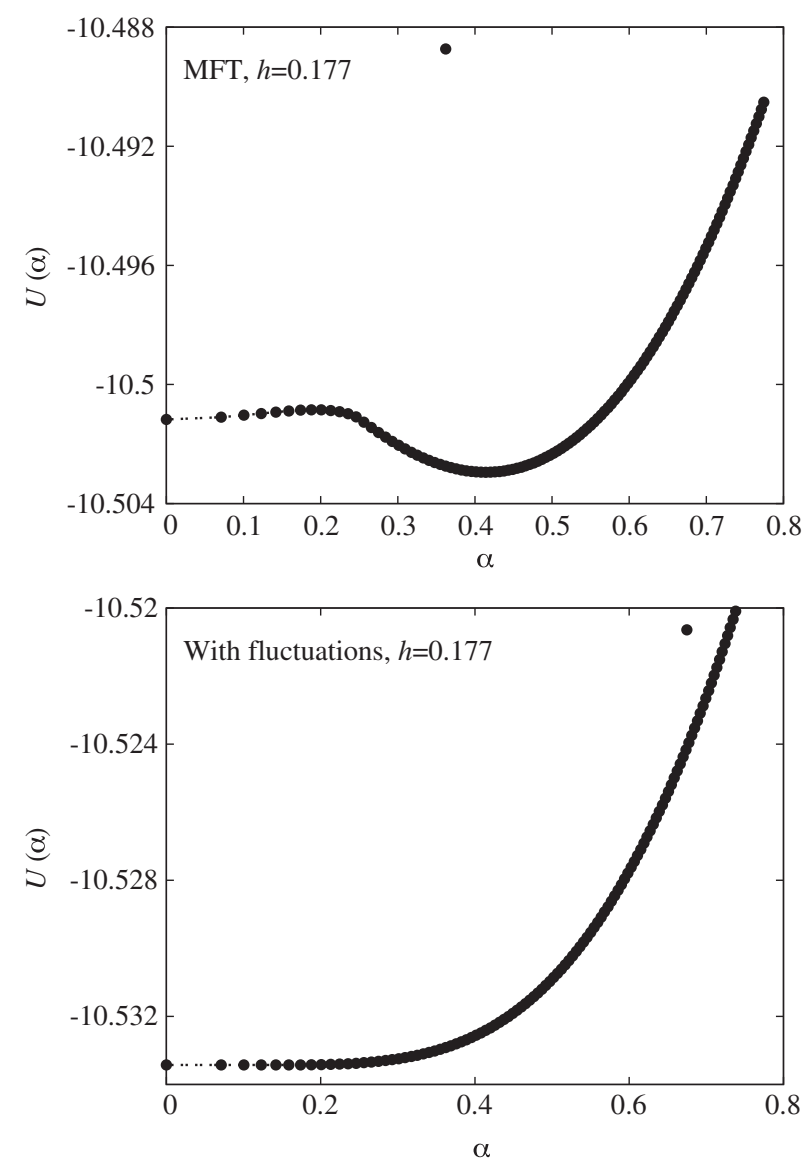

FIG. 6. Comparison of the zero-temperature potentials at the new QCP (black circles at $h=0.177, T=0$ in Fig. 3). Meanfield theory (top) incorrectly predicts a phase-separated fully gapped BCS plus excess fermion state with the global minimum at $\alpha_{0}=0.42$, which overshadows the Fermi surface mismatch. Fluctuations (bottom) smear out the curved region in the potential and, in fact, lead to a quantum phase transition with continuously onsetting superfluid order $\alpha_{0}$ slightly above zero. Numerical parameters and initial values of the $Z$ and $X$ factors are $g=-2$, $Z_{\Omega}^{\Lambda_{0}}=Z_{\mathbf{q}}^{\Lambda_{0}}=1$, and $X^{\Lambda_{0}}=0.2$. Further technical details are given in Appendix B.

excess fermion state and instead indicate a continuous quantum phase transition to a superfluid state with two Fermi surfaces, which could exist as an intermediate phase. Upon further increasing the interaction or reducing the imbalance $h$, this is expected to become a fully gapped BCS state with no Fermi surface after passing an intermediate state with one Fermi surface with enhanced density of states (when the black-dashed quasiparticle branch in Fig. 1 is pushed upwards by larger values of the gap $\alpha>h$ ) [22]. Note that the most significant renormalization of the potential $U(\rho)$ occurs relatively early in the flow (intermediate $\Lambda$ ), where the key effect is that regions with large curvature are smoothed out by quantum fluctuations. Figures 6 and 7 exhibit a comparison of the mean-field potential with its renormalized version at various points in the phase diagram.
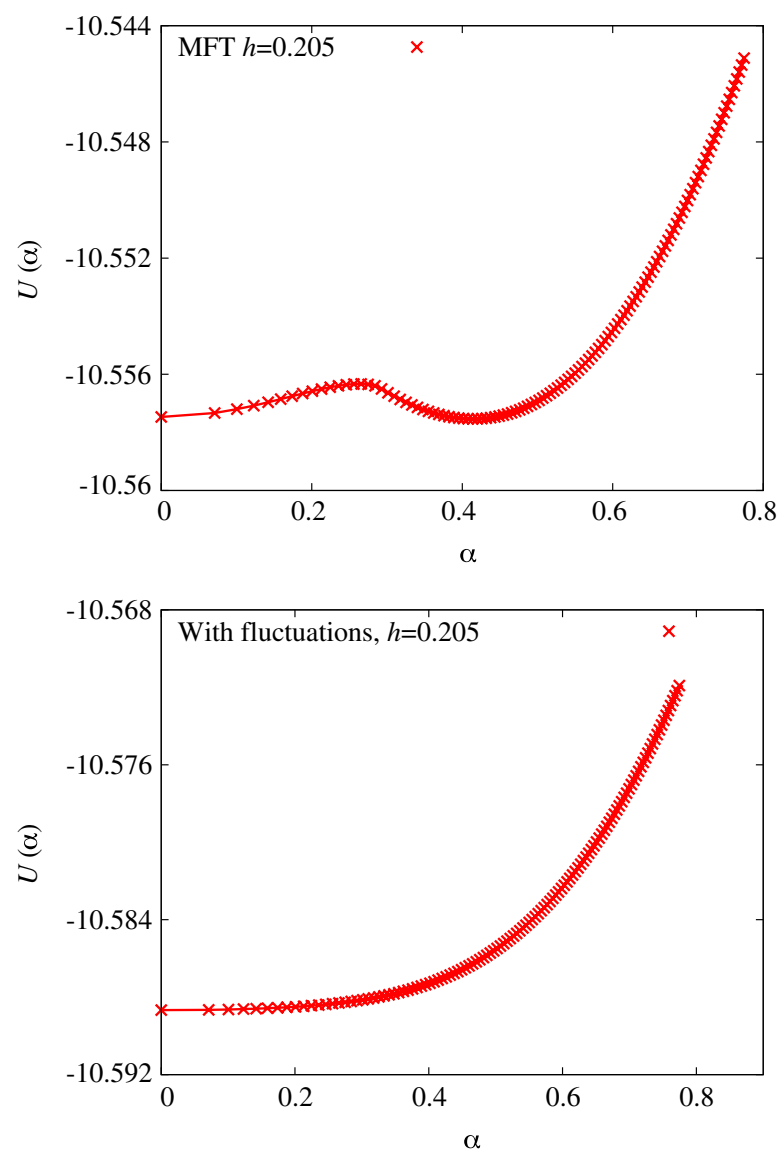

FIG. 7. Comparison of the zero-temperature potentials at the putative mean-field first-order transition (red cross at $h=0.205$, $T=0$ in Fig. 3). Mean-field theory (top) incorrectly predicts a first-order transition to a phase-separated fully gapped BCS plus excess fermion state with a second minimum at $\alpha_{0}=0.41$ degenerate to the $\alpha_{0}=0$ one, the former overshadowing the Fermi surface mismatch. Fluctuations (bottom), however, suppress the ordering tendencies and instead yield a symmetric state with $\alpha_{0}=0$. The other initial parameters are the same as in Fig. 6.

Let us observe that the above situation does not occur for sufficiently large absolute values of the coupling $g$, in which case fluctuations are not sufficiently potent to induce a QCP and the transition at $T=0$ remains first order also after renormalization. By tuning $g$, one goes between the two possibilities crossing a specific value of $g$, where the system displays a quantum tricritical point.

Here, we also note the reassuring feature of Eqs. (4) and (7) that at any finite $T$, a solution with a finite minimum $\alpha_{0}$, which would indicate spontaneous symmetry breaking, is not permissible. This is because of a logarithmic running of $\alpha_{0}$ from the Goldstone loop such that $\alpha_{0} \rightarrow 0$ for $\Lambda \rightarrow 0$ at finite $T$.

We close this section by noting that the ultimate fate of the intermediate gapless superfluid in two dimensions will also depend strongly on fermionic self-energy effects, such as those discussed Sec. IV and fluctuations in the shape of 
the Fermi surfaces, and the interplay with competing instabilities. An intriguing scenario [70] is the possibility of a merging of the two QCPs in Fig. 3 to form an isolated QCP at $(h=0, T=0)$, indicating the absence of any superfluid phase in the ground state. Resolution of such possibilities requires studying the interplay of the bosonic potential and single-particle, fermionic effects.

\section{NON-FERMI-LIQUID MECHANISM}

We now outline how the single-fermion properties are affected by order parameter fluctuations at the imbalanced superfluid quantum phase transition. To this end, we first write down the effective quantum field theory coupling the two mismatched Fermi surfaces to the (bosonic) Cooper pairing field containing one fermion from each Fermi surface. We then present a repeated scattering mechanism that is expected to reduce the lifetime of the quasiparticles around the Fermi surfaces leading to deviations from the Fermi liquid.

The relevant Lagrangian density contains the propagators of the two Fermion species,

$$
\mathcal{L}_{\bar{\psi} \psi \nu}=\bar{\psi}_{k \uparrow}\left(-i k_{\tau}+\xi_{\mathbf{k} \uparrow}\right) \psi_{k \uparrow}+\bar{\psi}_{k \downarrow}\left(-i k_{\tau}+\xi_{\mathbf{k} \downarrow}\right) \psi_{k \downarrow},
$$

with $\xi_{\mathbf{k} \sigma}$ fermion dispersions leading to two mismatched Fermi surfaces (Fig. 8), a complex-valued, dynamical Cooper pairing field,

$$
\mathcal{L}_{\phi^{*} \phi}=\phi_{q}^{*}\left(-X i q_{\tau}+Z_{\Omega} q_{\tau}^{2}+Z_{\mathbf{q}} \mathbf{q}^{2}+r\right) \phi_{q},
$$

whose leading frequency $\left(q_{\tau}\right)$ and momentum $(\mathbf{q})$ dependence is determined by the particle-particle bubble explained in Fig. 9. $r$ is the usual control parameter; here, we focus on the quantum critical point where $r=0$. The complex, linear frequency term determines the bare collective dynamics to have dynamical exponent $z=2$. Note that the Cooperon propagator Eq. (9) is only valid when frequencies $q_{\tau}$ and momenta $\mathbf{q}$ are small when compared to the Fermi energy. At higher scales, the bi-fermion character of the $\phi$ bosons results in a (decay) continuum and branch cuts in the complex plane, as per the logarithm of Eq. (C4); then the simple pole structure of Eq. (9) is

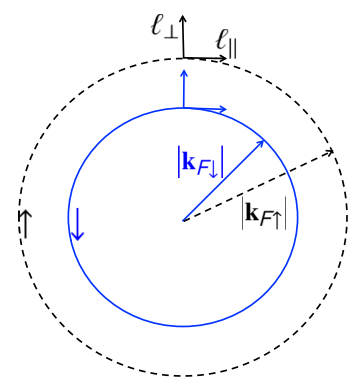

FIG. 8. Mismatched Fermi surfaces and local coordinate systems in momentum space.

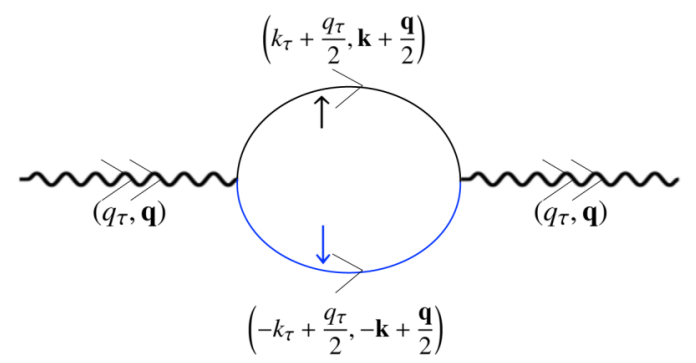

FIG. 9. Imbalanced particle-particle bubble that determines the collective Cooper pair dynamics (the $X$ and $Z$ factors) given in Eq. (9). Generalized to the superfluid phase, fermionic contractions of this form determine the initial values of the Goldstone and longitudinal propagators in the renormalization group flow of Eqs. (5) and (6). Wiggly line is the bosonic Cooper pair propagator, blue straight line is the spin-down fermion propagators, and black straight line is the spin-up fermion propagator. The cubic vertex connecting the three is the Yukawa coupling.

insufficient to describe the collective dynamics properly. Expanding this logarithm gives the expressions for the $X$ and $Z$ factors (see Appendix C).

The Cooper pairs are coupled to the pair of mismatched Fermi surfaces via a Yukawa coupling with strength $g$,

$\mathcal{L}_{\psi \phi}=g\left(\phi_{q} \bar{\psi}_{-k+(q / 2) \uparrow} \bar{\psi}_{k+(q / 2) \downarrow}+\phi_{q}^{*} \psi_{k+(q / 2) \downarrow} \psi_{-k+(q / 2) \uparrow}\right)$,

such that they can decay and recombine into fermions. The authors of Ref. [47] wrote down related critical field theories for Bose-Fermi mixtures with the aim of describing Fermi-Bose molecule formation of the fermions with fundamental bosons.

We now analyze the structure of the repeated scattering mechanism off finite $\left(q_{\tau}, \mathbf{q}\right)$ fluctuations at the quantum critical point. The single-scattering event of Fig. 10 is infrared finite because the respective, internal fermion line is always evaluated off shell, that is, away from its Fermi surface. Replacing the blue (spin-down) fermion line with a constant, the phase measure from the $q_{\tau}$ and $\mathbf{q}$ integration yields an (infrared) finite integral over the massless Cooper

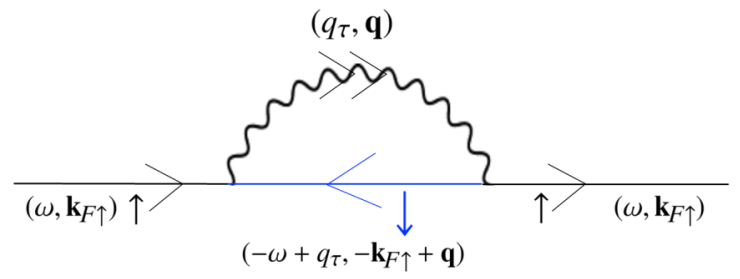

FIG. 10. Infrared finite one-loop contribution to the fermion self-energy. The internal (blue) fermion line is always evaluated off shell, that is, away from its Fermi surface. Note that in the different LOFF case, where the Cooperon carries finite momentum, this diagram can induce non-Fermi-liquid behavior. This case is further discussed in Sec. II. 
pair propagator with $z=2$ dynamics. The leading infrareddangerous scattering process for the frequency-dependent self-energy of the spin-up Fermi surfaces, $\Sigma_{\uparrow}\left(\omega, \mathbf{k}_{F \uparrow}\right)$, is a two-loop contraction shown in the top of Fig. 4:

$$
\begin{aligned}
\Sigma_{\uparrow}\left(\omega, \mathbf{k}_{F \uparrow}\right)= & g^{4} \int_{\boldsymbol{\ell}, \ell_{\tau}}\left\{\int_{\mathbf{q}, q_{\tau}}\left[G_{\downarrow}\left(-\omega+q_{\tau},-\mathbf{k}_{F \uparrow}+\mathbf{q}\right)\right]^{2}\right. \\
& \left.\times D\left(q_{\tau}, \mathbf{q}\right) D\left(q_{\tau}+\ell_{\tau}, \mathbf{q}+\boldsymbol{\ell}\right)\right\} \\
& \times G_{\uparrow}\left(\omega+\ell_{\tau}, \mathbf{k}_{F \uparrow}+\boldsymbol{\ell}\right) \\
& \equiv g^{4} \int_{\boldsymbol{\ell}, \ell_{\tau}}\left\{C\left(\ell_{\tau}, \boldsymbol{\ell}\right)\right\} G_{\uparrow}\left(\omega+\ell_{\tau}, \mathbf{k}_{F \uparrow}+\boldsymbol{\ell}\right),
\end{aligned}
$$

where we will view the function $C\left(\ell_{\tau}, \boldsymbol{\ell}\right)$ as a strongly frequency- and momentum-dependent intraspecies interaction. In local coordinate systems around the Fermi surfaces (cf. Fig. 8), the (on-shell) fermion propagator can be expressed as

$$
\begin{aligned}
& G_{\uparrow}\left(\omega+\ell_{\tau}, \mathbf{k}_{F \uparrow}+\boldsymbol{\ell}\right) \\
& =\frac{1}{-i\left(\omega+\ell_{\tau}\right)+v_{F \uparrow} \ell_{\perp}+\frac{1}{2 m_{\uparrow}}\left(\ell_{\perp}^{2}+\ell_{\|}^{2}\right)},
\end{aligned}
$$

with Fermi velocity $v_{F \uparrow}=\sqrt{\mu_{\uparrow} / 2 m_{\uparrow}}$. The $\ell_{\perp}^{2}$ term is subdominant in the infrared compared to the linear $v_{F \uparrow} \ell_{\perp}$ term. The Cooper pair propagator at criticality is

$$
D\left(q_{\tau}, \mathbf{q}\right)=\frac{1}{-i q_{\tau}+q_{\tau}^{2}+\mathbf{q}^{2}},
$$

where we may set the $X$ and $Z$ factors to unity here. Note that, in an effectively particle-hole symmetric situation (in symmetric shells around the Fermi surfaces), the complex linear frequency term must vanish and the bare dynamical exponent would be $z=1$. With disorder from trapping inhomogeneities or an additional species off which the Cooper pairs could scatter, one would have overdamped dynamics $\sim\left|q_{\tau}\right|[71]$.

Evaluating the spin-down fermions (the blue lines in the red box of Fig. 4) at the spin-up Fermi surface $\mathbf{k}_{F \uparrow}$ makes them off shell, and the infrared-dominant contribution of the dynamical intraspecies interaction then comes from the two Cooper pair propagators

$$
C_{\mathrm{IR}}\left(\ell_{\tau}, \boldsymbol{\ell}\right) \sim \int \frac{d^{2} \mathbf{q}}{(2 \pi)^{2}} \int \frac{d q_{\tau}}{2 \pi} D\left(q_{\tau}, \mathbf{q}\right) D\left(q_{\tau}+\ell_{\tau}, \mathbf{q}+\boldsymbol{\ell}\right),
$$

which, for example, is logarithmically singular with a bare $z=2$ power counting. The precise form of the effective single-species interaction mediated by density fluctuations and two critical Cooperons also needs to account for the nonanalyticities from pair-breaking effects from the logarithm of the Cooperon, Eq. (C4), when summing over higher-energy intermediate states as well as more precise experimental considerations, such as trapping potentials and the presence of disorder. Upon closing this singular interaction with the on-shell spin-up fermion propagator Eq. (12), we conclude that the lifetime of quasiparticles cannot have the Fermi liquid form [where the interactions are regular and IR finite and $\left.\operatorname{Im} \Sigma_{\uparrow}\left(\omega, \mathbf{k}_{\mathrm{F} \uparrow}\right) \sim \omega^{2}\right]$, but instead will receive anomalous corrections.

A similar mechanism was recently shown to induce nonFermi behavior in antiferromagnetic metals [52]. Strong non-Fermi-liquid behavior ("hot Fermi surfaces") and the total destruction of quasiparticles would require a strongcoupling resummation of the logarithmic singularities of Eq. (14) as found by Abrahams et al. [41] in the spinfermion model; such a resummation would effectively lead to power-law, long-ranged interaction as found by Bares and Wen [72].

Compared with the spin-density wave and Ising nematic quantum critical points in Fig. 2, the homogeneous imbalanced superfluid discussed in this paper is expected to feature warm Fermi surfaces leading to anomalous thermodynamics and transport but, in the absence of band crossings or intersections of the two Fermi surfaces, ultimately stable quasiparticles. At the hot spots in the spin-density wave case $(\operatorname{Im} \Sigma \sim \sqrt{\omega})$, or in the antinodal region of the nematic case $\left(\operatorname{Im} \Sigma \sim \omega^{2 / 3}\right)$, one has truly hot regions without any quasiparticles at all.

\section{CONCLUSION AND OUTLOOK ON EXPERIMENTS}

This paper has two main messages. First, we show that quantum fluctuations may favor the appearance of exotic superfluids in two-dimensional, imbalanced mixtures of fermionic atoms. Second, our results put imbalanced fermionic superfluids on the map of plain vanilla quantum phase transitions (Fig. 2) in two spatial dimensions exhibiting non-Fermi-liquid features. In light of the experimental progress in homogeneous trapping of fermionic atoms in reduced dimensionality, it is our hope that the results of mean-field approaches (applicable mostly to three-dimensional gases) will be carefully revisited in experiments and theory in two spatial dimensions.

Order parameter fluctuations, captured by solving a partial differential flow equation for the effective potential of the imbalanced Fermi gas, are shown to have dramatic beyond-mean-field effects on the zero-temperature phase diagram: for a range of parameters, a new, fluctuationinduced quantum critical point is found and Goldstone fluctuations are shown to destroy spontaneous symmetry breaking completely, in accordance with the MerminWagner theorem.

We argue that this case is qualitatively different from the more frequently studied case of fermions coupled to gauge field and the hot spot phenomenology at the onset of 
antiferromagnetic order in metals. Its key features are as follows.

(i) The collective mode(s) appears in the pairing channel spontaneously breaking the global U(1) symmetry. This leads to a single Goldstone mode whose fluctuations are shown to turn the mean-field phase-separated ground state into a homogeneous, strongly coupled imbalanced superfluid with gapless fermion excitations. This also leads to different features in the quantum critical field theory coupling two mismatched Fermi surfaces to the Cooperon. This Cooperon, for example, has a complex, linear time derivative that leads to a nontrivial coupling of Goldstone and longitudinal couplings in the superfluid phase. It may additionally be Landau damped from small disorder or trap inhomogeneities. Moreover, at the quantum critical point, one-loop vertex corrections renormalizing the Yukawa coupling vanish by particle conservation-these structural novelties make a comprehensive, diagrammatic analysis of the coupled Fermi-Bose theory an interesting topic for future research.

(ii) Non-Fermi-liquid physics around the Fermi surfaces appears although the Cooperon does not directly connect points or patches in the two mismatched Fermi surfaces. Repeated scattering processes should lead to warm Fermi surfaces with reduced, non-Fermi-liquid quasiparticle lifetimes.

(iii) The microscopic model of the parent compound is directly realizable in present-day experiments with ultracold mixtures of fermionic atoms. Techniques such as rf spectroscopy $[48,50]$ bear the potential to measure the reduced fermion lifetimes. The frequency-resolved rf signal is proportional to the overlap of a noninteracting fermion propagator from a noninteracting third level with the interacting fermion and, therefore, should be able to resolve the single-particle self-energy [73]. Furthermore, one can measure the frequencies of collective Rabi oscillations directly, which can also be related to the quasiparticle weight [17].

The QCPs of Fig. 3 should also have observable consequences such as anomalous thermodynamics [6] up to relatively, high, finite temperatures in the quantum critical regime, even in a scenario in which it gets "masked" by $p$-wave superfluidity at the lowest scales. Finally, the collective modes such as sound and the longitudinal "Higgs" mode can also be measured, for example, by time modulating the trapping potential and measuring the associated density response of the gas [74]. It would, therefore, be of great interest to generalize recent experiments with two-dimensional Fermi gases $[75,76]$ to Fermi mixtures [17] in the superfluid regime.

\section{ACKNOWLEDGMENTS}

We acknowledge helpful discussions with Bertrand Delamotte, Nicolas Dupuis, Matthias Punk, Subir Sachdev, Brian Swingle, Tarik Yefsah, and Martin Zwierlein. The research of P.S. was supported by the
DFG under Grant No. Str 1176/1-1, the NSF under Grant No. DMR-1103860, the John Templeton Foundation, the Center for Ultracold Atoms (CUA), and the Multidisciplinary University Research Initiative (MURI). P. J. acknowledges funding by the National Science Centre via Grant No. 2011/03/B/ST3/02638.

\section{APPENDIX A: LINKING THE FLOW TO FERMIONIC CORRELATIONS}

Her, we present the derivation of the mean-field effective potential Eq. (3) and the order parameter propagators Eq. (5), thereby providing a direct link of the order parameter flow to the underlying fermionic atoms.

We go to the thermodynamic limit and employ an action representation for Eq. (1). We perform a HubbardStratonovich transformation with the spin-singlet pairing field $\phi_{q}$ conjugate to the fermion bilinear

$$
\tilde{\phi}_{q}=g \int_{k} \psi_{k+(q / 2) \uparrow} \psi_{-k+(q / 2) \downarrow} .
$$

We employ the shorthand notation $\int_{k}=T \sum_{k_{\tau}} \int d^{2} \mathbf{k} /$ $(2 \pi)^{2}$ with fermionic (imaginary) Matsubara frequencies $k_{\tau}=2 \pi(n+1 / 2) T$, with $n$ integer running from $-\infty$ to $\infty$. Their bosonic pendant is conventionally denoted by $q_{\tau}=2 \pi n T$. Later, we will take the zero-temperature limit $T \rightarrow 0$ and focus on ground-state properties. The resulting functional integral is over $\psi, \bar{\psi}$, and $\phi$, with the new bare action

$$
\begin{aligned}
\mathcal{S}[\psi, \bar{\psi}, \phi]= & \int_{k} \sum_{\sigma=\uparrow, \downarrow} \bar{\psi}_{k \sigma}\left(-i k_{\tau}+\xi_{\mathbf{k} \sigma}\right) \psi_{k \sigma}-\int_{q} \phi_{q}^{*} \frac{1}{g} \phi_{q} \\
& +\int_{k, q}\left(\bar{\psi}_{-k+(q / 2) \downarrow} \bar{\psi}_{k+(q / 2) \uparrow} \phi_{q}\right. \\
& \left.+\psi_{k+(q / 2) \uparrow} \psi_{-k+(q / 2) \downarrow} \phi_{q}^{*}\right),
\end{aligned}
$$

where $\phi^{*}$ is the complex conjugate of $\phi$, while $\psi$ and $\bar{\psi}$ are algebraically independent Grassmann variables. Note that as $g<0$, the bosonic mass term $-1 / g$ is positive.

We first shift $\phi_{q}$ by a nonfluctuating (static in time and homogenous in position space) field $\Delta$ :

$$
\phi_{q} \rightarrow(2 \pi)^{2} \delta_{\mathbf{q}, 0} \frac{\delta_{q_{\tau, 0}}}{T} \Delta+\phi_{q},
$$

where the factors of $(2 \pi)^{2}$ are conventional and translate to the correct volume normalization factors in position space. Equation (A2) is recast as $\mathcal{S}=\mathcal{S}_{b}+\mathcal{S}_{f}$, with

$$
\begin{aligned}
\mathcal{S}_{b}= & \frac{(2 \pi)^{2}}{T}|\Delta|^{2}\left(\frac{-1}{g}\right)+\left(\frac{-1}{g}\right)\left(\Delta \phi_{0}^{*}+\Delta^{*} \phi_{0}\right) \\
& +\int_{q} \phi_{q}^{*} \phi_{q}\left(\frac{-1}{g}\right) .
\end{aligned}
$$


We employ a convenient Nambu matrix notation for the fermionic part:

$$
\begin{aligned}
\mathcal{S}_{f}= & \int_{k}\left(\bar{\psi}_{k \uparrow} \psi_{-k \downarrow}\right) \overbrace{\left(\begin{array}{cc}
-i k_{\tau}+\xi_{\mathbf{k} \uparrow} & -\Delta \\
-\Delta^{*} & -i k_{\tau}-\xi_{-\mathbf{k} \downarrow}
\end{array}\right)}^{\equiv \mathcal{S}_{f}^{(2)}[\Delta]}\left(\begin{array}{c}
\psi_{k \uparrow} \\
\bar{\psi}_{-k \uparrow}
\end{array}\right) \\
& +\int_{k, q}\left(\bar{\psi}_{k+(q / 2) \uparrow} \psi_{-k+(q / 2) \downarrow}\right)\left(\begin{array}{cc}
0 & -\phi_{q} \\
-\phi_{q}^{*} & 0
\end{array}\right)\left(\begin{array}{c}
\psi_{k+(q / 2) \uparrow} \\
\bar{\psi}_{-k+(q / 2) \uparrow}
\end{array}\right) .
\end{aligned}
$$

This action is quadratic in the Grassmann fermion fields, and we can perform the Gaussian functional integral. After expanding the resulting tr $\log$ to second order in $\phi_{q}$, we obtain

$$
\begin{aligned}
\mathcal{S}[\phi]= & \int_{q} \phi_{q}^{*}\left(\frac{-1}{g}+K(q)\right) \phi_{q}+\int_{q} \phi_{q}^{*} \phi_{-q}^{*} \frac{L(q)}{2} \\
& +\int_{q} \phi_{q} \phi_{-q} \frac{L^{*}(q)}{2}-\frac{(2 \pi)^{2}}{T} \frac{1}{g}|\Delta|^{2}-\operatorname{tr} \ln \beta \mathcal{S}_{f}^{(2)}[\Delta] \\
& +\left(\frac{-1}{g}\right)\left(\Delta \phi_{0}^{*}+\Delta^{*} \phi_{0}\right) \\
& -\left(\phi_{0}^{*} \int_{k} F_{f \uparrow \downarrow}(k)+\phi_{0} \int_{k} F_{f \uparrow \downarrow}^{*}(k)\right),
\end{aligned}
$$

where the tr goes over Nambu indices, frequencies, and momenta. The terms in the last line cancel, if $\Delta$ fulfills the saddle-point condition or gap equation,

$$
\Delta=-g \int_{k} F_{f \uparrow \downarrow}(k),
$$

which is what one gets from differentiating the second line of Eq. (A6) with respect to $\phi_{0}^{*}$. Here, we have used the symmetric normal $(K)$ and anomalous $(L)$ particle-particle pair propagators,

$$
\begin{aligned}
K(q) & =-\int_{k} G_{f \uparrow}\left(k+\frac{q}{2}\right) G_{f \downarrow}\left(-k+\frac{q}{2}\right), \\
L(q) & =\int_{k} F_{f \uparrow \downarrow}\left(k+\frac{q}{2}\right) F_{f \downarrow \uparrow}\left(-k+\frac{q}{2}\right),
\end{aligned}
$$

and their single-particle constituents,

$$
\begin{aligned}
G_{f \uparrow}(k) & =\frac{-i k_{\tau}-\xi_{-\mathbf{k} \downarrow}}{\left(i k_{\tau}-\xi_{\mathbf{k} \uparrow}\right)\left(-i k_{\tau}-\xi_{-\mathbf{k} \downarrow}\right)+|\Delta|^{2}}, \\
F_{f \uparrow \downarrow}(k) & =\frac{\Delta}{\left(i k_{\tau}-\xi_{\mathbf{k} \uparrow}\right)\left(-i k_{\tau}-\xi_{-\mathbf{k} \downarrow}\right)+|\Delta|^{2}} .
\end{aligned}
$$

To bring out the physics of Goldstone and longitudinal fluctuations, we now perform a change of variables in Eq. (A6), $\mathcal{S}[\phi] \rightarrow \mathcal{S}[\sigma, \pi]$, with

$$
\phi_{q}=\frac{\sigma_{q}+i \pi_{q}}{\sqrt{2}}, \quad \phi_{q}^{*}=\frac{\sigma_{-q}-i \pi_{-q}}{\sqrt{2}},
$$

where $\pi$ is the transversal Goldstone field variable and $\sigma$ the radial Higgs field variable. $\Delta$ is chosen real and denoted by $\alpha$ such that

$$
|\Delta|^{2}=\frac{\alpha^{2}}{2} \equiv \rho .
$$

The action is obtained as

$$
\begin{aligned}
\mathcal{S}[\sigma, \pi, \alpha]= & \int_{q} \frac{\sigma_{-q} \sigma_{q}}{2}\left[-g^{-1}+K(q)+\operatorname{Re}\{L(q)\}\right]+\int_{q} \frac{\pi_{-q} \pi_{q}}{2}\left[-g^{-1}+K(q)-\operatorname{Re}\{L(q)\}\right] \\
& +\int_{q} \frac{\sigma_{-q} \pi_{q}}{2}(i K(q)-i \operatorname{Im}\{L(q)\})+\frac{\pi_{-q} \sigma_{q}}{2}(-i K(q)-i \operatorname{Im}\{L(q)\})+\frac{(2 \pi)^{2}}{T} U[\alpha],
\end{aligned}
$$

with $U[\alpha]$ the local effective potential given in Eq. (3) that survives the $q \rightarrow 0$ mean-field limit. The mean-field phase diagram of Fig. 3 is determined by the field value $\alpha$ that globally minimizes the potential

$$
\Omega^{0}(\mu, h, r, T)=\min _{\alpha}\{U[\alpha]\} .
$$

The $q \neq 0$ terms composed of fermionic pair propagators represent phase and amplitude fluctuations about the meanfield saddle point.

The mass terms of the radial and Goldstone propagators can be obtained from derivatives of $U[\alpha]$. For the Goldstone propagator, one gets $\frac{1}{\alpha} U^{\prime}[\alpha] \equiv \frac{1}{\alpha} \frac{\partial U}{\partial \alpha}=\frac{-1}{g}-\frac{1}{\alpha} \int_{k} F_{f \uparrow \downarrow}(k)=\frac{-1}{g}+K(0)-L(0)$,

where the second line uses the explicit expression of the pair propagators in Eq. (A8). The Goldstone mass is zero if $U^{\prime}\left[\alpha_{0}\right]=0$ or, equivalently, $\alpha_{0}$ fulfills the saddle-point condition or gap equation. For the radial propagator, one gets

$$
\begin{aligned}
U^{\prime \prime}[\alpha] & =\frac{\partial^{2} U}{\partial \alpha^{2}}=\frac{1}{\alpha} \frac{\partial U}{\partial \alpha}+2 \operatorname{Re}\{L(0)\} \\
& =\frac{-1}{g}+K(0)+\operatorname{Re}\{L(0)\},
\end{aligned}
$$


where we use Eq. (A14). $U^{\prime \prime}\left[\alpha_{0}\right]$ is finite providing the mass gap to the longitudinal fluctuations. We finally rewrite Eq. (A12) using Eqs. (A14) and (A15) to obtain

$$
\begin{aligned}
\mathcal{S}[\sigma, \pi, \alpha]= & \int_{q} \frac{\sigma_{-q} \sigma_{q}}{2}\left\{Q_{\sigma \sigma}(q ; \alpha)+U^{\prime}[\alpha]\right\}+\frac{(2 \pi)^{2}}{T} U[\alpha] \\
& +\int_{q} \frac{\pi_{-q} \pi_{q}}{2}\left\{Q_{\pi \pi}(q ; \alpha)+\frac{1}{\alpha} U^{\prime}[\alpha]\right\} \\
& +\int_{q}\left[\frac{\sigma_{-q} \pi_{q}}{2} Q_{\sigma \pi}(q ; \alpha)+\frac{\pi_{-q} \sigma_{q}}{2} Q_{\sigma \pi}(-q ; \alpha)\right],
\end{aligned}
$$

with the momentum behavior of the propagators

$$
\begin{aligned}
& Q_{\sigma \sigma}(q)=K(q)+\operatorname{Re}\{L(q)\}-[K(0)+\operatorname{Re}\{L(0)\}], \\
& Q_{\pi \pi}(q)=K(q)-\operatorname{Re}\{L(q)\}-[K(0)-\operatorname{Re}\{L(0)\}], \\
& Q_{\sigma \pi}(q)=i K^{\text {odd }}(q)-i \operatorname{Im}\{L(q)\},
\end{aligned}
$$

where the superscript "odd" projects out frequency-and momentum components that transform odd under $q \rightarrow-q$. Clearly, $Q_{\sigma \sigma}(q=0)=Q_{\pi \pi}(q=0)=Q_{\sigma \pi}(q=0)=0$.

Equations (A17) tie the renormalization group analysis for order parameter fluctuations [Eq. (5)] to the underlying fermionic correlations.

\section{APPENDIX B: DETAILS OF THE RENORMALIZATION GROUP FLOW}

Here, we give some details about the applied renormalization group procedure. We restrict the momentum integrations to $|\mathbf{k}|<3.5\left|\mathbf{k}_{F}\right|=3.5 \times \sqrt{2}$, mimicking the UV regularization of an attractive lattice Hubbard model at low filling fractions. In our computations, the initial momentum scale $\Lambda_{0}$ at which we start the flow is a free parameter. Generally, this should be the momentum scale below which order parameter fluctuations are expected to kick in, and here we take $\Lambda_{0}=\sqrt{2} \sim\left|\mathbf{k}_{F}\right|$, attempting to account for an entire class of particle-hole and higher-order diagrams, which are formally omitted within our truncation. These are expected to reduced ordering scales significantly [77].

The applied procedure is an adapted variant of a leadingorder calculation in the functional renormalization group derivative expansion. The next-to-leading order would also treat the $Z$ and $X$ factors as functions of $\rho$ and $\Lambda$. Our main focus here is the zero-temperature flows leading to the phase diagram in Fig. 3. For this situation, a reliable approximation [78] neglects the order parameter flow of $Z$ and $X$ altogether and leaves their initial values at $\Lambda=\Lambda_{0}$ unchanged from the values guided by the form of the fermionic theory, Eqs. (A17), and attempts to account for particle-hole fluctuations and higher-order corrections. Here, we choose $Z_{\Omega}^{\Lambda_{0}}=Z_{\mathbf{q}}^{\Lambda_{0}}=1$ and $X^{\Lambda_{0}}=0.2$.

\section{APPENDIX C: IMBALANCED PARTICLE-PARTICLE BUBBLE}

The imbalanced particle-particle bubble drawn in Fig. 9, whose low-energy expansion leads to the Cooper pair propagator [Eqs. (9) and (13)], is defined as

$$
\Pi(q)=\Pi_{\mathrm{pp}}\left(i q_{\tau}, \mathbf{q}\right)-\Pi_{\mathrm{pp}}(0,0),
$$

with

$$
\begin{aligned}
\Pi_{\mathrm{pp}}\left(i q_{\tau}, \mathbf{q}\right)= & -\int_{-\infty}^{\infty} \frac{d k_{\tau}}{2 \pi} \int_{-\infty}^{\infty} \frac{d^{2} \mathbf{k}}{(2 \pi)^{2}} \\
& \times G_{\uparrow}(k+q / 2) G_{\downarrow}(-k+q / 2),
\end{aligned}
$$

and

$$
G_{\sigma}(k)=\frac{1}{-i k_{\tau}+\xi_{\mathbf{k} \sigma}}=\frac{1}{-i k_{\tau}+\frac{\mathbf{k}^{2}}{2 m_{\sigma}}-\mu_{\sigma}} .
$$

We can successively perform the integrations first $k_{\tau}$, then $k_{x}$, and $k_{y}$ last to find

$$
\Pi(q)=\frac{-1}{4 \pi} \log \left[1+\frac{Q(q)}{2 \mu}\right],
$$

where $\mu=\frac{1}{2}\left(\mu_{\uparrow}+\mu_{\downarrow}\right)$ and

$$
Q(q)=Q\left(q_{\tau}, \mathbf{q}\right)=i q_{\tau}-\mathbf{q}^{2}\left[\frac{1}{4 m_{r}}-\frac{m_{r}}{16}\left(\frac{1}{m_{\uparrow}}-\frac{1}{m_{\downarrow}}\right)^{2}\right] .
$$

The logarithm in Eq. (C4) will, in general, lead to branch cuts in the complex (frequency plane). For energies and momenta smaller than the effective binding energy $2 \mu$, we can expand the logarithm to obtain Eq. (9) with

$$
\begin{gathered}
X=\frac{1}{8 \pi \mu}, \quad Z_{\Omega}=\frac{1}{32 \pi \mu^{2}}, \\
Z_{\mathbf{q}}=\frac{1}{32 \pi \mu m_{r}}\left[1-\frac{m_{r}^{2}}{4}\left(\frac{1}{m_{\uparrow}}-\frac{1}{m_{\downarrow}}\right)^{2}\right],
\end{gathered}
$$

where we further used the reduced mass $1 / m_{r}=$ $\frac{1}{2}\left(1 / m_{\uparrow}+1 / m_{\downarrow}\right)$.

[1] L. D. Landau, Theory of Fermi Liquids, Sov. Phys. JETP 3, 920 (1957); Oscillations in a Fermi Liquid, 5, 101 (1957).

[2] P. Nozières, Theory of Interacting Fermi Systems (Benjamin, Amsterdam, 1964).

[3] A. J. Schofield, Non-Fermi Liquids, Contemp. Phys. 40, 95 (1999).

[4] C. M. Varma, Z. Nussinov, and W. van Saarloos, Singular or Non-Fermi Liquids, Phys. Rep. 361, 267 (2002).

[5] T. Schaefer and K. Schwenzer, Non-Fermi Liquid Effects in QCD at High Density, Phys. Rev. D 70, 054007 (2004). 
[6] H. v. Loehneysen, A. Rosch, M. Vojta, and P. Woelfle, Fermi-Liquid Instabilities at Magnetic Quantum Phase Transitions, Rev. Mod. Phys. 79, 1015 (2007).

[7] P. Coleman and A. J. Schofield, Quantum Criticality, Nature (London) 433, 226 (2005).

[8] S. Sachdev and B. Keimer, Quantum Criticality, Phys. Today 64, No. 2, 29 (2011).

[9] D. S. Greywall, Specific Heat of Normal Liquid ${ }^{3} \mathrm{He}$, Phys. Rev. B 27, 2747 (1983).

[10] D. Vollhardt and P. Woelfle, The Superfluid Phases Of Helium 3 (Taylor \& Francis, London, 2003).

[11] I. Bloch, J. Dalibard, and W. Zwerger, Many-Body Physics with Ultracold Gases, Rev. Mod. Phys. 80, 885 (2008).

[12] B. DeMarco and D. S. Jin, Onset of Fermi Degeneracy in a Trapped Atomic Gas, Science 285, 1703 (1999).

[13] Z. Hadzibabic, S. Gupta, C. Stan, C. Schunck, M. Zwierlein, K. Dieckmann, and W. Ketterle, Fiftyfold Improvement in the Number of Quantum Degenerate Fermionic Atoms, Phys. Rev. Lett. 91, 160401 (2003).

[14] M. Koehl, H. Moritz, T. Stöferle, K. Günter, and T. Esslinger, Fermionic Atoms in Three-Dimensional Optical Lattice: Observing Fermi Surfaces, Dynamics, and Interactions, Phys. Rev. Lett. 94, 080403 (2005).

[15] M. Zwierlein, A. Schirotzek, C. H. Schunck, W. Ketterle, Fermionic Superfluidity with Imbalanced Spin Populations, Science 311, 492 (2006).

[16] G. B. Partridge, W. Li, R. I. Kamar, Y.-a. Liao, R. G. Hulet, Pairing and Phase Separation in a Polarized Fermi Gas, Science 311, 503 (2006).

[17] C. Kohstall, M. Zaccanti, M. Jag, A. Trenkwalder, P. Massignan, G. M. Bruun, F. Schreck, and R. Grimm, Metastability and Coherence of Repulsive Polarons in a Strongly Interacting Fermi Mixture, Nature (London) 485, 615 (2012).

[18] W. V. Liu and F. Wilczek, Interior Gap Superfluidity, Phys. Rev. Lett. 90, 047002 (2003).

[19] G. Sarma, On the Influence of a Uniform Exchange Field Acting on the Spins of the Conduction Electrons in a Superconductor, J. Phys. Chem. Solids 24, 1029 (1963).

[20] P. F. Bedaque, H. Caldas, and G. Rupak, Phase Separation in Asymmetrical Fermion Superfluids, Phys. Rev. Lett. 91, 247002 (2003).

[21] S.-T. Wu and S. Yip, Superfluidity in the Interior-Gap States, Phys. Rev. A 67, 053603 (2003).

[22] W. V. Liu, F. Wilczek, and P. Zoller, Spin-Dependent Hubbard Model and a Quantum Phase Transition in Cold Atoms, Phys. Rev. A 70, 033603 (2004).

[23] A. Bulgac, M. M. Forbes, and A. Schwenk, Induced P-Wave Superfluidity in Asymmmetric Fermi Gases, Phys. Rev. Lett. 97, 020402 (2006).

[24] M. M. Parish, F. M. Marchetti, A. Lamacraft, and B. D. Simons, Polarized Fermi Condensates with Unequal Masses: Tuning the Tricritical Point, Phys. Rev. Lett. 98, 160402 (2007).

[25] L. He and P. Zhuang, Phase Diagram of a Cold Polarized Fermi Gas in Two Dimensions, Phys. Rev. A 78, 033613 (2008).

[26] S. Pilati and S. Giorgini, Phase Separation in a Polarized Fermi Gas at Zero Temperature, Phys. Rev. Lett. 100, 030401 (2008).
[27] A. E. Feiguin and M. P. A. Fisher, Exotic Paired States with Anisotropic Spin-Dependent Fermi Surfaces, Phys. Rev. Lett. 103, 025303 (2009).

[28] R. B. Diener and M. Randeria, BCS-BEC Crossover with Unequal-Mass Fermions, Phys. Rev. A 81, 033608 (2010).

[29] L. Radzihovsky and D. E. Sheehy, Imbalanced FeshbachResonant Fermi Gases, Rep. Prog. Phys. 73, 076501 (2010).

[30] A. Kujawa-Cichy and R. Micnas, Stability of Superfluid Phases in the 2D Spin-Polarized Attractive Hubbard Model, Europhys. Lett. 95, 37003 (2011).

[31] D. E. Sheehy, Polarized Superfluids near Their Tricritical Point, Phys. Rev. A 79, 033606 (2009).

[32] S. Yin, J.-P. Martikainen, and P. Torma, Fulde-Ferrel States and Berezinskii-Kosterlitz-Thouless Phase Transition in Two-Dmensional Imbalanced Fermi Gases, Phys. Rev. B 89, 014507 (2014).

[33] A. M. Clogston, Upper Limit for the Critical Field in Hard Superconductors, Phys. Rev. Lett. 9, 266 (1962).

[34] K. Binder, Theory of First-Order Phase Transitions, Rep. Prog. Phys. 50, 783 (1987).

[35] H. v. Löhneysen, A. Rosch, M. Vojta, and P. Wölfle, FermiLiquid Instabilities at Magnetic Quantum Phase Transitions, Rev. Mod. Phys. 79, 1015 (2007).

[36] D. Belitz, T. R. Kirkpatrick, and T. Vojta, How Generic Scale Invariance Influences Quantum and Classical Phase Transitions, Rev. Mod. Phys. 77, 579 (2005).

[37] R. L. Greenblatt, M. Aizenman, and J. L. Lebowitz, Rounding of First-Order Transitions in Low-Dimensional Quantum Systems with Quenched Disorder, Phys. Rev. Lett. 103, 197201 (2009).

[38] P. Jakubczyk, J. Bauer, and W. Metzner, Finite Temperature Crossovers near Quantum Tricritical Points in Metals, Phys. Rev. B 82, 045103 (2010).

[39] M. A. Metlitski and S. Sachdev, Quantum Phase Transitions of Metals in Two Spatial Dimensions: II. Spin Density Wave Order, Phys. Rev. B 82, 075128 (2010).

[40] J. Lee, P. Strack, and S. Sachdev, Quantum Criticality of Reconstructing Fermi Surfaces in Antiferromagnetic Metals, Phys. Rev. B 87, 045104 (2013).

[41] E. Abrahams, J. Schmalian, and P. Woelfle, Strong Coupling Theory of Heavy Fermion Criticality, arXiv: 1303.3926.

[42] V. Oganesyan, S. A. Kivelson, and E. Fradkin, Quantum Theory of Nematic Fermi Fluid, Phys. Rev. B 64, 195109 (2001).

[43] W. Metzner, D. Rohe, and S. Andergassen, Soft Fermi Surfaces and Breakdown of Fermi-Liquid Behavior, Phys. Rev. Lett. 91, 066402 (2003).

[44] S.-S. Lee, Low-Energy Effective Theory of Fermi Surface Coupled with U(1) Gauge Field in $2+1$ Dimensions, Phys. Rev. B 80, 165102 (2009).

[45] G. v. Gersdorff and C. Wetterich, Nonperturbative Renormalization Flow and Essential Scaling for the KosterlitzThouless Transition, Phys. Rev. B 64, 054513 (2001).

[46] At finite temperatures, the proposed truncation is not sufficient to describe the relevant BKT physics. Nevertheless, it is clear that also for $T>0$ the flow at large $\Lambda$ is dominated by the quantum terms and the initial evolution of the potential proceeds exactly along the same pattern. The additional thermal contributions are proportional to $T$ and 
become relevant exclusively at large RG time (low $\Lambda$ ) where the structures of $U(\alpha)$ typical to a first-order transition had been smeared out by the flow. It is, therefore, a plausible scenario that a finite BKT transition line (drawn in Fig. 3) will connect the two newly found QCPs.

[47] S. Powell, S. Sachdev, and H. P. Büchler, Depletion of BoseEinstein Condensate in Bose-Fermi Mixtures, Phys. Rev. B 72, 024534 (2005).

[48] J. T. Stewart, J. P. Gaebler, and D. S. Jin, Using Photoemission Spectroscopy to Probe a Strongly Interacting Fermi Gas, Nature (London) 454, 744 (2008).

[49] A. J. A. James and A. Lamacraft, Non-Fermi-Liquid Fixed Point for an Imbalanced Gas of Fermions in $1+\epsilon$ Dimensions, Phys. Rev. Lett. 104, 190403 (2010).

[50] Z. Lan, G. M. Bruun, and C. Lobo, Quasiparticle Lifetime in Ultracold Fermionic Mixtures with Density and Mass Imbalance, Phys. Rev. Lett. 111, 145301 (2013).

[51] K. R. Patton and D. E. Sheehy, Induced p-Wave Superfluidity in Strongly Interacting Imbalanced Fermi Gases, Phys. Rev. A 83, 051607(R) (2011).

[52] S. A. Hartnoll, D. M. Hofman, M. A. Metlitski, and S. Sachdev, Quantum Critical Response at the Onset of Spin-Density-Wave Order in Two-Dimensional Metals, Phys. Rev. B 84, 125115 (2011).

[53] Y. Liao, A. S. C. Rittner, T. Paprotta, W. Li, G. B. Partridge, R. G. Hulet, S. K. Baur, and E. J. Mueller, Spin-Imbalance in a One-Dimensional Fermi Gas, Nature (London) 467, 567 (2010).

[54] M. J. Wolak, B. Gremaud, R. T. Scalettar, and G. G. Batrouni, Pairing in Two-Dimensional Fermi Gas with Population Imbalance, Phys. Rev. A 86, 023630 (2012).

[55] D. Roscher, J. Braun, and J. E. Drut, Inhomogeneous Phases in One-Dimensional Mass- and Spin-Imbalanced Fermi Gases, arXiv:1311.0179.

[56] D. E. Sheehy and L. Radzihovsky, BEC-BCS Crossover, Phase Transitions, and Phase Separation in Polarized Resonantly Paired Superfluids, Ann. Phys. (Amsterdam) 322, 1790 (2007).

[57] R. M. Lutchyn, M. Dzero, and V. M. Yakovenko, Spectroscopy of the Soliton Lattice Formation in QuasiOne-Dimensional Fermionic Superfluids with Population Imbalance, Phys. Rev. A 84, 033609 (2011).

[58] P. Jakubczyk, W. Metzner, and H. Yamase, Turning a FirstOrder Quantum Phase Transition Continuous by Fluctuations: General Flow Equations and Application to d-Wave Pomeranchuk Instability, Phys. Rev. Lett. 103, 220602 (2009).

[59] C. Langmack, M. Barth, W. Zwerger, and E. Braaten, Clock Shift in a Strongly Interacting Two-Dimensional Fermi Gas, Phys. Rev. Lett. 108, 060402 (2012).

[60] G. Bertaina and S. Giorgini, BCS-BEC Crossover in a TwoDimensional Fermi Gas, Phys. Rev. Lett. 106, 110403 (2011).

[61] P. Bloom, Two-Dimensional Fermi Gas, Phys. Rev. B 12, 125 (1975).
[62] M. Randeria, J.-M. Duan, and L.-Y. Shieh, Bound States, Cooper Pairing, and Bose Condensation in Two Dimensions, Phys. Rev. Lett. 62, 981 (1989).

[63] A. J. Leggett, in Modern Trends in the Theory of Condensed Matter, edited by A. Pekalski and R. Przystawa (Springer, Berlin, 1980).

[64] F. Pistolesi, C. Castellani, C. D. Castro, and G. C. Strinati, Renormalization Group Approach to the Infrared Behavior of a Zero-Temperature Bose System, Phys. Rev. B 69, 024513 (2004).

[65] P. Strack, R. Gersch, and W. Metzner, Renormalization Group Flow for Fermionic Superfluids at Zero Temperature, Phys. Rev. B 78, 014522 (2008).

[66] C. Wetterich, Exact Evolution Equation for the Effective Potential, Phys. Lett. B 301, 90 (1993).

[67] J. Berges, N. Tetradis, and C. Wetterich, Nonperturbative Renormalization Flow in Quantum Field Theory and Statistical Physics, Phys. Rep. 363, 223 (2002).

[68] R. B. Diener, R. Sensarma, and M. Randeria, Quantum Fluctuations in the Superfluid State of the BCS-BEC Crossover, Phys. Rev. A 77, 023626 (2008).

[69] B. Obert, C. Husemann, and W. Metzner, Low-Energy Singularities in the Ground State of Fermionic Superfluids, Phys. Rev. B 88, 144508 (2013).

[70] H. Yamase, P. Jakubczyk, and W. Metzner, Nematic Quantum Criticality Without Order, Phys. Rev. B 83, 125121 (2011).

[71] A. Del Maestro, B. Rosenow, and S. Sachdev, Theory of Pair-Breaking Superconductor-Metal Transition in Nanowires, Ann. Phys. (Amsterdam) 324, 523 (2009).

[72] P.-A. Bares and X.-G. Wen, Breakdown of the Fermi Liquiddue to Long-Range Interactions, Phys. Rev. B 48, 8636 (1993).

[73] R. Haussmann, M. Punk, and W. Zwerger, Spectral Functions and rf Response of Ultracold Fermionic Atoms, Phys. Rev. A 80, 063612 (2009).

[74] E. Vogt, M. Feld, B. Fröhlich, D. Pertot, M. Koschorreck, and M. Köhl, Scale-Invariance and Viscosity of a TwoDimensional Fermi Gas, Phys. Rev. Lett. 108, 070404 (2012).

[75] M. Feld, B. Fröhlich, E. Vogt, M. Koschorreck, and M. Köhl, Observation of a Pairing Pseudogap in a Two-Dimensional Fermi Gas, Nature (London) 480, 75 (2011).

[76] B. Fröhlich, M. Feld, E. Vogt, M. Koschorreck, W. Zwerger, and M. Köhl, Radio-Frequency Spectroscopy of a Strongly Interacting Two-Dimensional Fermi Gas, Phys. Rev. Lett. 106, 105301 (2011).

[77] L. P. Gorkov and T. K. Melik-Barkhudarov, Contribution to the Theory of Superfluidity in an Imperfect Fermi Gas, Sov. Phys. JETP 13, 1018 (1961).

[78] On the other hand, if taken into account, anomalous scaling of the propagator occurs exclusively at the transition and only once the fixed point is attained (at low $\Lambda$ ). Therefore, our approximation should have no impact on our conclusions. 